\title{
Recent progress in high pressure metrology in Europe
}

\author{
Wladimir Sabuga ${ }^{1}$, Dominik Pražák ${ }^{2, a}$ and Thierry Rabault ${ }^{3}$ \\ ${ }^{1} \mathrm{PTB}$, Braunschweig, Germany \\ ${ }^{2}$ CMI, Brno, Czech Republic \\ ${ }^{3}$ LNE, Paris, France
}

\begin{abstract}
Five European national metrology institutes in collaboration with a university, a research institute and five industrial companies are working on a joint research project within a framework of the European Metrology Research Programme aimed at development of 1.6 GPa primary and 1.5 GPa transfer pressure standards. Two primary pressure standards were realised as pressure-measuring multipliers, each consisting of a low pressure and a high pressure (HP) piston-cylinder assembly (PCA). A special design of the HP PCAs was developed in which a tungsten carbide cylinder is supported by two thermally shrunk steel sleeves and, additionally, by jacket pressure applied to the outside of the outer sleeve. Stress-strain finite element analysis (FEA) was performed to predict behaviour of the multipliers and a pressure generation system. With FEA, the pressure distortion coefficient was determined, taking into account irregularities of the piston-cylinder gap. Transfer pressure standards up to $1.5 \mathrm{GPa}$ are developed on the basis of modern $1.5 \mathrm{GPa}$ pressure transducers. This project shall solve a discrepancy between the growing needs of the industry demanding precise traceable calibrations of the high pressure transducers and the absence of adequate primary standards for pressures higher than $1 \mathrm{GPa}$ in the European Union today.
\end{abstract}

\section{Introduction}

New high pressure technologies such as autofrettage, hydroforming and isostatic pressing are being widely developed and used in the car industry, diesel engineering, vessel production for the petrochemical and pharmaceutical industries, manufacturing water cutting machines, new material fabrication and, recently, for food sterilisation. With these new technologies, the quality and lifetime of products and the efficiency of processes can be increased, material and energy resources saved, and the emission of pollutants reduced. New transducers for measuring pressures up to $1.5 \mathrm{GPa}$ have recently been used in new high pressure applications. Several European consultancy groups predict that high pressure sensing will grow in the near future. However, no suitable standards for pressures higher than $1 \mathrm{GPa}$ are currently available in the EU with which the modern pressure transducers could be adequately calibrated.

In addition to a lack of primary pressure standards, there are no reliable, sufficiently investigated transfer pressure standards for pressures up to $1.5 \mathrm{GPa}$. In industry, the lack of high pressure standards means that the measurement properties of pressure transducers calibrated at lower pressures must be extrapolated to higher pressures. This approach provides only a vague estimation of pressure outside of the transducers' calibrated range, with negative consequences for the efficiency and safety of the industrial processes. Without a calibration service provided for companies using highpressure technologies, companies cannot fulfil their quality assurance and safety requirements, which hinders any expected technology improvements if the process pressure increases.

To close this gap, five European national metrology institutes (NMIs), PTB (Germany), CMI (Czech Republic), METAS (Switzerland), LNE (France) and SMU (Slovakia), as well as the Technical University Clausthal (Germany), in cooperation with six industrial and research companies, have launched a joint research project (JRP) "High pressure metrology for industrial applications" within the European Metrology Research Programme (EMRP) [1, 2]. The JRP goes beyond the state of the art by creating two primary pressure standards for pressures up to $1.6 \mathrm{GPa}$ with a relative expanded uncertainty as low as $5 \cdot 10^{-4}$, which is sufficiently low to calibrate modern $1.5 \mathrm{GPa}$ pressure transducers, and by developing transfer standards and calibration methods for the range 0.1 to $1.5 \mathrm{GPa}$ based on high pressure transducers. Finally, an internationally recognised, routinely available and affordable pressure calibration service up to 1.5 GPa should be established in Europe.

\footnotetext{
$\bar{a}$ Corresponding author: dprazak@ cmi.cz
} 


\section{Research contents}

The primary 1.6 GPa pressure standards are realised as pressure-measuring multipliers, each comprising 2 coupled low pressure (LP) and high pressure (HP) pistoncylinder assemblies (PCAs) equipped with a pressure generation and transmission system. The transfer standard will include a group of pressure transducers with upper ranges between 0.5 and 1.5 GPa. Various research activities are required for their realisation and metrological characterisation:

- Finite Element Analysis (FEA) to determine optimal design of the LP and HP PCAs, to predict behaviour of HP components such as tubing, connectors, valves and lenses and to find out solutions for a reliable tight connection of the HP components.

- Strain gauge measurements on commercial HP tubing, connections and valves as well as tests of their performance at pressures up to $1.6 \mathrm{GPa}$.

- Hardness measurements on steel materials of the HP components of the pressure transmission system.

- Measurement of the elastic constants of tungsten carbide and steel materials used for the PCAs and the pressure generation system.

- Measurement of density and viscosity versus pressure of potential pressure-transmitting liquids to be used in the new primary and transfer HP standards. Test of the liquids' stability when subject to pressures up to 1.6 GPa.

- Development, manufacture and test of a $1.6 \mathrm{GPa}$ pressure generation, transmission and control system.

- Investigation of $1.5 \mathrm{GPa}$ pressure transducers and design development of the HP transducers-based transfer standards.

- Dimensional measurements of the PCAs' geometry and, in particular, of the piston-cylinder gap profile.

- Metrological characterisation of the new 1.6 GPa pressure standards using FEA and validation of the theoretical results by cross-float experiments.

- Assembly and characterisation of 1.5 GPa transfer pressure standards by a comparison against the national pressure standards of the NMIs - JRP participants.

\section{Progress and results}

The main progress was achieved in the application of FEA to study stress-strain, elastic-plastic deformation and contact processes in the HP components of the pressure balances, calculation of the pressure distortion coefficient and prediction of the piston fall rate. With the new FEA methodology, novel $1.6 \mathrm{GPa}$ pressure-measuring multipliers could be modelled, analysed and built. Elastic constants of steels and numerous tungsten carbides (WC) were measured by strain gauges and resonant ultrasound spectroscopy (RUS). The hardness of high pressure components was determined and the effect of thermal treatment was investigated. Density and viscosity of potential pressure-transmitting liquids were measured at atmospheric pressure and room temperature. The stability of these liquids after they had been pressurised up to
1.6 GPa was investigated. Commercial HP components were tested at pressures up to $1.6 \mathrm{GPa}$, and their modifications implemented. An HP pressure transducersbased 1.5 GPa transfer standard was assembled and tested.

\subsection{Pressure-measuring multipliers}

The operation principle of a pressure-measuring multiplier is explained in figure 1.

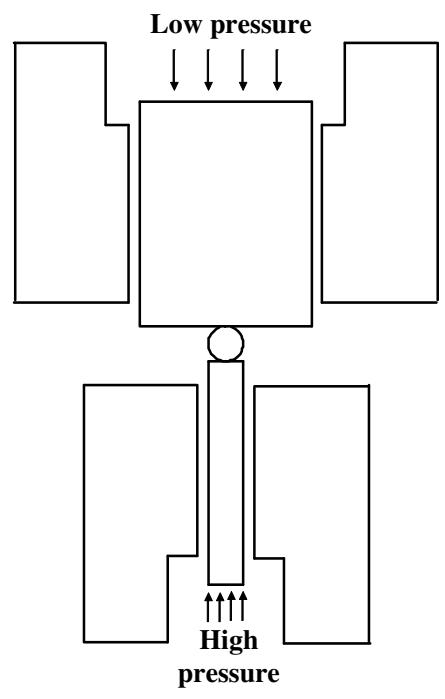

Figure 1. Operation principle of a pressure multiplier.

The multiplier includes low pressure $\left(p_{\mathrm{L}}\right)$ and high pressure $\left(p_{\mathrm{H}}\right)$ PCAs which have significantly different effective areas. The LP and HP PCAs are axially aligned and their pistons are mechanically coupled. Both LP and HP pistons are unsealed in the cylinders and are rotated, which, due to the lubrication effect, avoids mechanical friction between the pistons and cylinders. Consequently, in the absence of other forces, the forces due to pressures $p_{\mathrm{H}}$ and $p_{\mathrm{L}}$ on the pistons are balanced when the ratio of pressures is equal to the ratio of the effective areas of the LP and HP PCAs, $A_{\mathrm{HP}}$ and $A_{\mathrm{LP}}$ :

$$
p_{\mathrm{H}} / p_{\mathrm{L}}=A_{\mathrm{HP}} / A_{\mathrm{LP}}
$$

The high pressure, $p_{\mathrm{H}}$, can thus be determined by accurately measuring $p_{\mathrm{L}}$ and by knowing the exact ratio of $A_{\mathrm{HP}}$ to $A_{\mathrm{LP}}$, also called the multiplying ratio $\left(K_{\mathrm{M}}\right)$. The principle of the pressure-measuring multipliers has been in use at least since the 1930s and is utilised in current practice, for example in the $1.5 \mathrm{GPa}$ national pressure standard of Russia, VNIIFTRI [3]. A $1 \mathrm{GPa}$ pressure multiplier has been commercially offered since the late 1980s and is used by NMIs and calibration laboratories as a secondary or transfer standard [4].

Two new pressure multipliers intended as primary pressure standards for the $1.6 \mathrm{GPa}$ range with an expected relative expanded uncertainty as low as $5 \cdot 10^{-4}$ have been jointly developed and built by PTB and Fluke Calibration, USA [5]. 


\subsubsection{Design}

In the newly developed multiplier, the nominal effective areas of the LP and HP PCAs were chosen to be $1 \mathrm{~cm}^{2}$ and $5 \mathrm{~mm}^{2}$, respectively. These are dimensions for which production technology is well established and which result in a pressure ratio of 1:20. Thus, pressure $p_{\mathrm{H}}=1.6 \mathrm{GPa}$ on the HP side of the multiplier is reached at $p_{\mathrm{L}}=80 \mathrm{MPa}$ which can be easily generated and measured with high accuracy.

The design of the new multiplier has specific features which distinguish it from that of the former multipliers. First, to avoid plastic deformation and to guarantee stability of the effective areas, the components of the LP and HP PCAs are made of tungsten carbide with $6 \%$ or $10 \%$ (HP piston) cobalt (WC-Co) instead of steel as used in [3]. Since the tensile strength of the tungsten carbide is limited to roughly $0.7 \mathrm{GPa}$, the HP cylinder can be operated at the maximum pressure of $1.6 \mathrm{GPa}$ only if it is supported from outside. Thanks to a special design of the multipliers, a compressive load is established on the outside of the HP tungsten carbide cylinder which prevents rupture when the pressure inside the cylinder exceeds the material tensile strength. In [4], this is accomplished by fitting a sleeve around the cylinder. In order to extend this to $1.6 \mathrm{GPa}$ in the new multiplier, two sleeves, each made of chrome/nickel/molybdenum steel, are successively assembled onto the tungsten carbide HP cylinder by means of thermal shrink fits. In addition, the HP cylinder with the two sleeves is set into a jacket which allows a jacket pressure $\left(p_{\mathrm{j}}\right)$ to be applied to the lateral surface of the outer sleeve, which thus additionally compensates the tensile stress in the cylinder (figure 2).

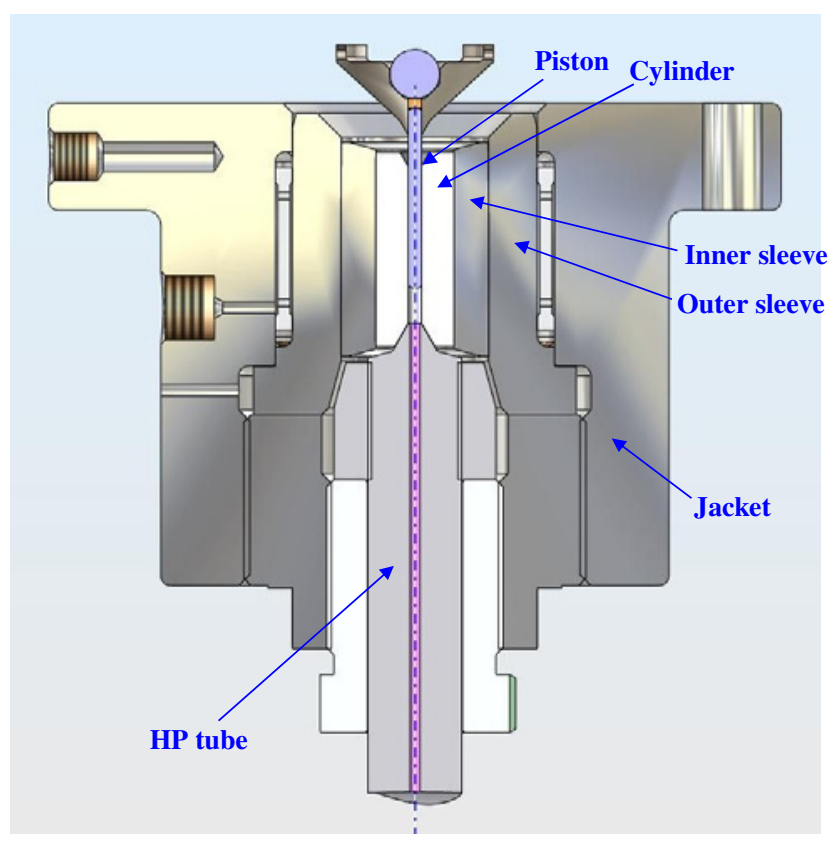

Figure 2. HP PCA in the mounting post.

The HP PCA is designed to be operated in controlled clearance (CC) mode with $p_{\mathrm{j}}$ typically equal to $25 \%$ of $p_{\mathrm{H}}$, at which the pressure distortion coefficient $(\lambda)$ of the PCA should be around zero. In addition, the HP PCA may be operated with variable $p_{\mathrm{j}}$ in order to adjust the piston fall rate $\left(v_{\mathrm{f}}\right)$ and the PCA sensitivity, if necessary, as well as to study $\lambda$ experimentally by measuring dependences of $v_{\mathrm{f}}$ and $A_{\mathrm{HP}}$ on $p_{\mathrm{j}}$. The optimal shape of the inner sleeve was determined by FEA as described in the next section.

The LP PCA was designed while keeping in mind the requirement to have sufficiently low fluid flow through the piston-cylinder gap. This requirement results from the relatively large effective area of the LP PCA compared to the area of a pressure balance PCA maintaining and measuring $p_{\mathrm{L}}$. To limit the flow rate and optimize performance, the principle of negative free deformation was applied. In the LP PCA design, the LP cylinder is surrounded by a sleeve with a conical taper on the inside surface and $p_{\mathrm{L}}$ is applied to the outside surface of the sleeve. The LP sleeve has a sliding fit on the cylinder and is positioned so that its smallest diameter is located where the cylinder pressure is maximal. In the absence of pressure, the sleeve produces no stress on the cylinder. As pressure increases, loading the cylinder from inside and the sleeve from outside, the sleeve first comes into contact with the cylinder in the region where the pressure in the piston-cylinder gap is maximal. In this way, a variable outside load of the cylinder is created that optimally compensates the radial distortion of the cylinder produced by the inner pressure. With this variable outside load distribution a nearly linear pressure distribution in the LP PCA gap is achieved.

\subsubsection{FEA}

To analyse the feasibility of the pressure multipliers' operation up to $1.6 \mathrm{GPa}$ and to optimise the dimensions of the PCAs' components, they were modelled using FEA. The modelling at PTB was performed using the FEA software package ANSYS. The FEA included large deflection, contact and plastic capabilities, the latter required for the tube connected to the HP PCA. First, all parts were modelled with their material properties and assumed geometries. For the HP PCA, the shrinking of the inner and then of the outer sleeve on the tungsten carbide cylinder, and connection of the HP tube to the HP cylinder was modelled.

The cylinder to inner sleeve shrink fit was performed first, using nominal geometry. The deformation of the outer surface of the inner sleeve after this step was noted. The inner to outer sleeve shrink fit was performed second, using the resulting cylinder/inner sleeve combination with the outer sleeve nominal geometry. The shrink fit of the taper in the inner sleeve was accomplished in the same manner as the other shrink fit surfaces. The amount of contact of the surfaces was determined iteratively in the analysis, a step performed automatically by the FEA software. Three inner sleeve outside shapes were numerically tested in their effect on the stress, pressure distribution in the piston-cylinder gap and $\lambda$.

The connection of the HP tube to the cylinder and the deformation of the tube under pressure were studied. A tube tip angle of $59.5^{\circ}$ and matching cylinder cone angle of $60^{\circ}$ were selected. The tube was moved into the 
cylinder to establish contact along the whole length of the cylinder cone and a pressure of 1.6 GPa was applied.

Surface loads in various combinations were applied. The loads included $50 \%$ or $100 \%$ of maximum measurement pressure on relevant surfaces, a linear and, alternatively, constant pressure distribution in the pistoncylinder gap, as well as a jacket pressure on the outer surface of the HP PCA sleeve and an LP on the outer surface of the LP PCA sleeve. After each load step, radial deformation, radial and tangential stresses were extracted. The stress and strain distributions were analysed in relation to the ultimate tensile strength and the elastic limit of the cylinder, sleeve, and HP tube materials. These properties together with Young's modulus $(E)$ and Poisson's ratio $(\mu)$ accurately measured by the RUS and the strain gauge method as described in 3.2.1, were used in the FEA.

The FEA results demonstrated that the double shrink will to a great extent compensate for the stress produced by the internal pressure of $1.6 \mathrm{GPa}$. For a linear pressure distribution in the gap, the maximum residual stress produced by the shrinking and the internal pressure would be about $400 \mathrm{MPa}$, which could be withstood by the WC cylinder even in the absence of jacket pressure. However, in order to minimize the risk of cylinder rupture, jacket pressure is expected to be applied in all normal system operations.

Figure 3 presents the FEA model of the HP PCA and tangential stresses in the PCA components at $p_{\mathrm{H}}=1.6 \mathrm{GPa}$, a linear pressure distribution from $1.6 \mathrm{GPa}$ to zero along the piston-cylinder gap and $p_{\mathrm{j}}=0.4 \mathrm{GPa}$.

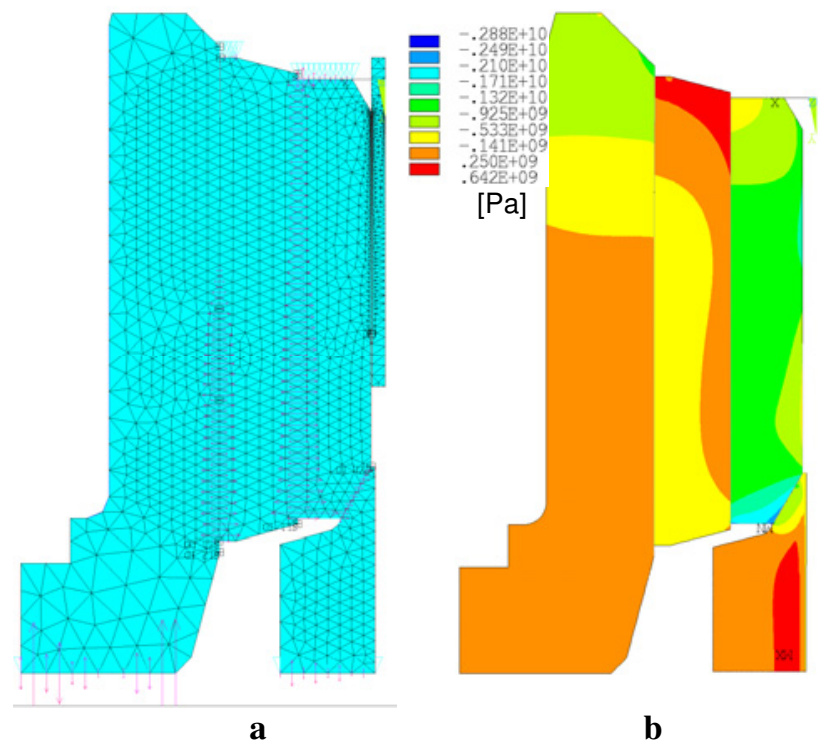

Figure 3. FEA model of HP PCA (a) and tangential stress distribution in it at $p_{\mathrm{H}}=1.6 \mathrm{GPa} \& p_{\mathrm{j}}=0.4 \mathrm{GPa}(\mathrm{b})$.

The FEA calculations for the HP PCAs at the maximum measurement pressure of $1.6 \mathrm{GPa}$ and the jacket pressure of $400 \mathrm{MPa}$ show that the radial and tangential stress distributions are smooth and without any significant concentrations, the cylinder is subject only to compressive stresses, and the sleeves remain within the elastic limit. These results for the HP PCA indicate that the design is not at risk for cylinder rupture nor instability of the effective area due to plastic deformation in the sleeves. The calculations also confirm the necessity of having two sleeves on the HP cylinder in order to achieve the required cylinder compression when the temperature for the thermal shrink is kept below the tempering temperature of the sleeve material.

The analysis of the tube shows that only a small portion of the tube near the centre line is subject to plastic deformation. In the tapered part of the tube, in the sections where the tube is not supported by the cylinder, the region of plastic deformation does not exceed 1/3 of the tube cross section. These results indicate a reliable connection between the HP PCA and tube at pressures up to $1.6 \mathrm{GPa}$.

In a similar manner as the HP PCA, an FEA of the LP PCA was performed at $p_{\mathrm{L}}=80 \mathrm{MPa}$ and a linear pressure distribution from $p_{\mathrm{L}}$ to zero was applied to the inside of the cylinder with $p_{\mathrm{L}}$ applied to the outside of the sleeve surrounding the cylinder. The primary objective was to minimise the radial distortions at the cylinder inside and thus the fluid flow rate. It was found out that with an optimal taper on the inside of the sleeve, the radial distortions of the cylinder do not exceed $0.1 \mu \mathrm{m}$ at any point of the cylinder bore. Without the sleeve and in the free deformation (FD) mode, they would reach $1 \mu \mathrm{m}$ at the gap entrance.

Combining the structural FEA of the HP PCA with a hydrodynamic analysis of its piston-cylinder gap, $\lambda$ and $v_{\mathrm{f}}$ were calculated using the iterative method described in [6]. As a pressure-transmitting medium, two liquids were considered: di(2)-ethyl-hexyl-sebacate (DHS) at $p_{\mathrm{H}} \leq 0.5 \mathrm{GPa}$ and polydiethylsiloxan PES-1 for $p_{\mathrm{H}} \leq 1.6 \mathrm{GPa}$, whose properties are presented in 3.2.3. With DHS, calculations were performed in FD mode to provide target values of $v_{\mathrm{f}}$ for optimal piston-cylinder gap widths to be achieved in the piston-cylinder production process. With PES-1, both FD and CC modes were analysed.

In the FEA, the PCAs were treated as axisymmetric with gap profiles determined by dimensional measurements as described in 3.1.3.

\subsubsection{Dimensional properties}

As known from former FEA studies, the results of hydrodynamic modelling strongly depend on the initial real gap profile between the undistorted piston and the cylinder [6]. In particular, information about the cylinder bore profile near the exit is important because the gap in this region becomes the narrowest under high pressure and therefore has a strong effect on the pressure distribution, $v_{\mathrm{f}}$ and $\lambda$. To take this into account, dimensional measurements were performed on the two HP cylinders. They included straightness measurements in the outlet region of the cylinder bore along 4 generatrix lines separated by $45^{\circ}$. The results for opposite generatrices $\left(0^{\circ}\right.$ and $180^{\circ}, 45^{\circ}$ and $225^{\circ}$, and so on) were averaged and are shown for the two cylinders in figure 4 . 


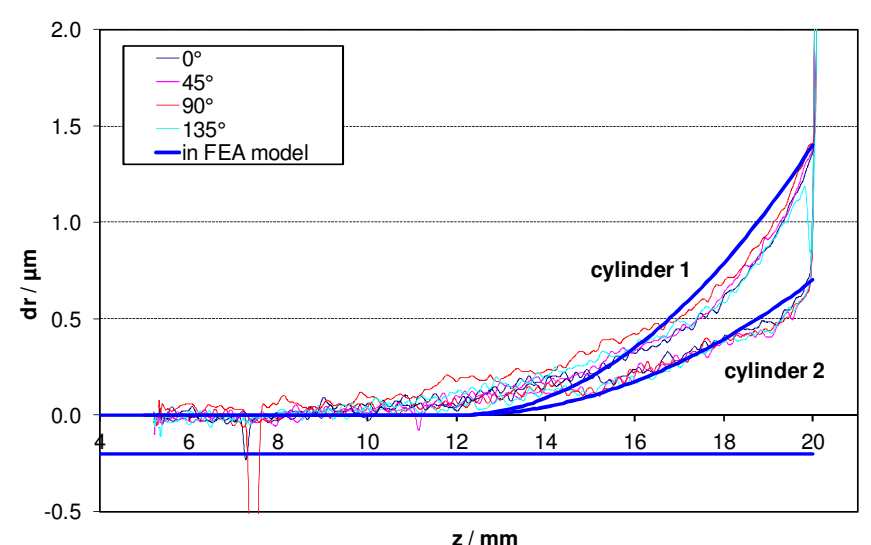

Figure 4. Piston-cylinder gap near the outlet for a perfect piston and real dimensions of cylinders 1 and 2 .

For FEA calculations, where the PCAs are treated as axisymmetric, all gap profiles were averaged and approximated by analytical functions which are also presented in figure 4. The piston and the cylinder bore, apart from the gap exit, were considered ideally cylindrical. Different gap widths $(h)$ were analysed. Figure 4 presents the case in which $h$ was equal to $0.2 \mu \mathrm{m}$.

\subsubsection{Predicted metrological properties}

The results of the piston fall rate calculations for $h=(0.2-0.5) \mu \mathrm{m}, \mathrm{FD}$ and CC operation modes, DHS and PES-1 liquids are shown in figure 5.

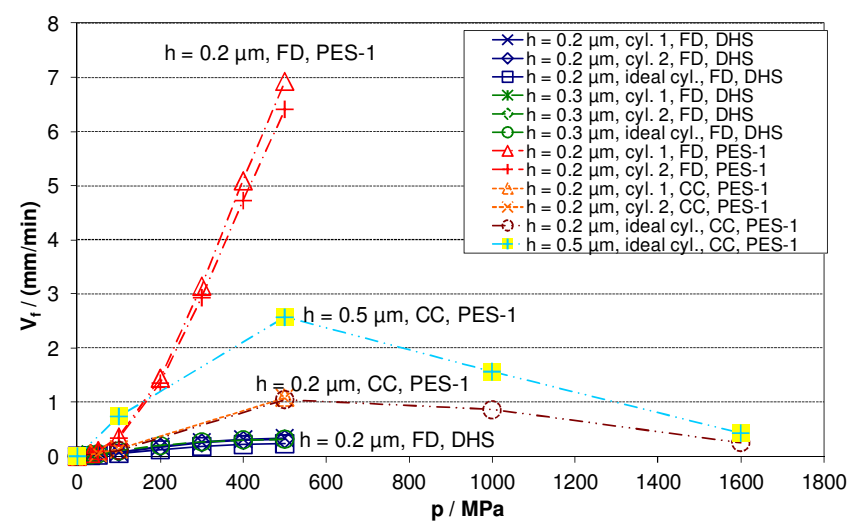

Figure 5. Piston fall rate calculated for different gap sizes, profiles and liquids.

Even with the smallest technologically feasible gap of $0.2 \mu \mathrm{m}, v_{\mathrm{f}}$ is too high when PES-1 and the FD mode are used. The largest gap considered, $0.5 \mu \mathrm{m}$, combined with the $\mathrm{CC}$ mode produces acceptable $v_{\mathrm{f}}$ at $p_{\mathrm{H}}>1 \mathrm{GPa}$, but rates that are still too high below $1 \mathrm{GPa}$. Surprisingly, the difference between the piston fall rates for $0.2 \mu \mathrm{m}$ and $0.5 \mu \mathrm{m}$ gaps in the pressure range (1 to 1.6) $\mathrm{GPa}$ is not as large as would be expected from the undistorted gap's theory. With the FEA results for $v_{\mathrm{f}}$, the range $h=(0.3-$ $0.4) \mu \mathrm{m}$ was found to be optimal. With $h=0.4 \mu \mathrm{m}$, a target $v_{\mathrm{f}}$ of $(0.068$ to 0.073$) \mathrm{mm} / \mathrm{min}$ was defined as achieved at $32 \mathrm{MPa}$ in control measurements when fitting the pistons to the cylinders. At $500 \mathrm{MPa}$ with DHS and in
FD mode, this gap width leads to $v_{\mathrm{f}}=(0.57-$ $0.61) \mathrm{mm} / \mathrm{min}$.

With the same gap sizes, profiles and liquids as used for $v_{\mathrm{f}}$ calculation, the pressure distortion coefficient of the HP PCA, $\lambda_{\mathrm{H}}$, was determined which is shown in figure 6 .

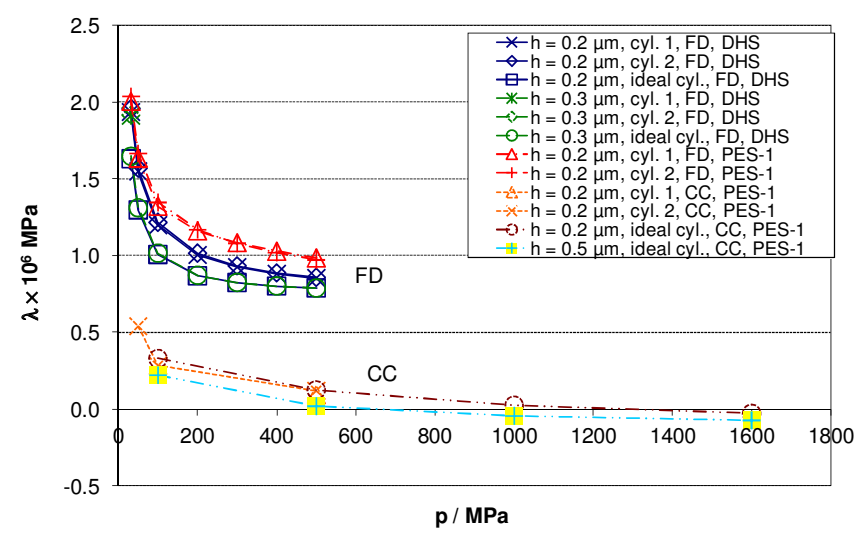

Figure 6. Pressure distortion coefficient of the HP PCA calculated for different gap sizes, profiles and liquids.

The dependence $\lambda_{\mathrm{H}}(p)$ becomes nonlinear towards smaller pressure. In contrast to $v_{\mathrm{f}}, \lambda_{\mathrm{H}}$ is less sensitive to the gap profile and liquid properties, but is mainly affected by the operation mode. At $500 \mathrm{MPa}$, the difference between $\lambda_{\mathrm{H}}$ in FD and CC mode is about $0.88 \cdot 10^{-6} \mathrm{MPa}^{-1}$. The effect of the gap and liquid is about $0.18 \cdot 10^{-6} \mathrm{MPa}^{-1}$ in FD and only $0.11 \cdot 10^{-6} \mathrm{MPa}^{-1}$ in $\mathrm{CC}$ mode.

For the LP PCA, its pressure distortion coefficient, $\lambda_{\mathrm{L}}$ - in the range $p_{\mathrm{L}}=[0 ; 25] \mathrm{MPa}$ corresponding to $p_{\mathrm{H}}=[0$; $500] \mathrm{MPa}$ - was preliminarily determined (without having exact dimensional information about the sliding sleeve) as lying between ( 0.87 to 0.89$) \times 10^{-6} \mathrm{MPa}^{-1}$.

The multiplying ratio of the multiplier $\left(K_{\mathrm{M}}\right)$ depends on pressure according to

$$
K_{\mathrm{M}}=K_{\mathrm{M}, 0} \times\left[1+\lambda_{K \mathrm{M}} \cdot\left(p_{\mathrm{H}}-p_{\mathrm{T}}\right)\right],
$$

where $K_{\mathrm{M}, 0}$ is $K_{\mathrm{M}}$ at $p_{\mathrm{L}}=0, \lambda_{K \mathrm{M}}$ is the pressure dependence coefficient of the multiplier and $p_{\mathrm{T}}$ is the tare pressure produced on the HP side of the multiplier by the masses loading the HP piston (HP and LP pistons, piston coupler, etc.), i.e. high pressure existing in the absence of low pressure $\left(p_{\mathrm{L}}=0\right)$. $\lambda_{K \mathrm{M}}$ depends on the distortion coefficients of the HP and LP PCAs as follows:

$$
\lambda_{K \mathrm{M}}=-\lambda_{\mathrm{H}}+\lambda_{\mathrm{L}} \times p_{\mathrm{L}} /\left(p_{\mathrm{H}}-p_{\mathrm{T}}\right)
$$

With $\lambda_{\mathrm{H}}$ and $\lambda_{\mathrm{L}}$ reported above, the distortion coefficient of the multipliers at $p_{\mathrm{H}}=500 \mathrm{MPa}$ is equal to about $\lambda_{K \mathrm{M}}=-0.05 \cdot 10^{-6} \mathrm{MPa}^{-1}$.

\subsubsection{Experimental results}

Very first tests of the multipliers were performed by Fluke at pressures (100 to 500) MPa on the HP side and (5 to 25) MPa on the LP side of the multipliers using two piston gauges as a reference, with DHS as a pressure transmitting liquid and at $p_{\mathrm{j}}=0.25 \cdot p_{\mathrm{H}}[5]$. 
Multiplying ratios were determined in a crossfloat. The pistons were kept within $\pm 2.5 \mathrm{~mm}$ around their middle working position. They were rotated by a DC motor at approximately $10 \mathrm{rpm}$. A hydraulic pressure controller was used to set $p_{\mathrm{j}}$. Tare pressure $p_{\mathrm{T}}$ was measured at $p_{\mathrm{L}}=0$ for each multiplier assembly using a pressure monitor with an uncertainty of approximately $2 \mathrm{kPa}$ $(k=2)$. It was equal to (2.918 and 2.926) MPa for the two multipliers.

A crossfloat, using the drop rate method, was performed between the two piston gauges with multiplier either 1 or 2 in between the two piston gauges at $p_{\mathrm{H}}=(100,200,300,400,500,500,400,300,200$, 100) $\mathrm{MPa}$ in a total of four runs. The multiplying ratio was determined at each point as shown in figure 7 .

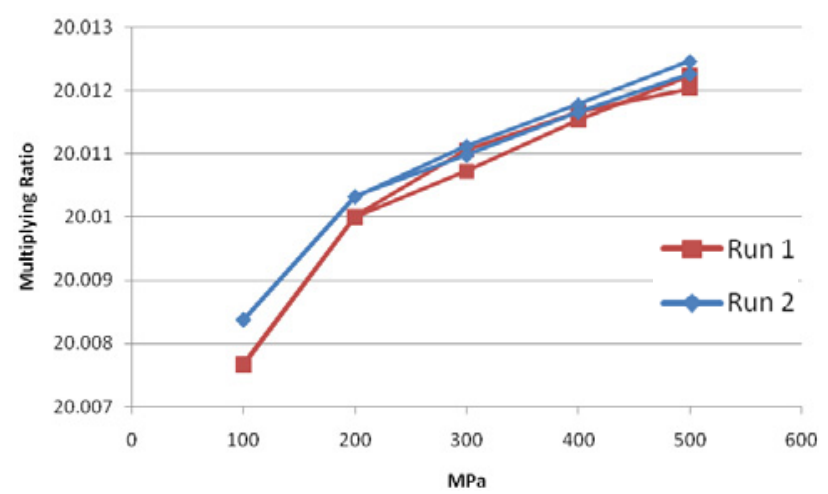

Figure 7. Multiplying ratio vs. high pressure corrected for tare pressure.

The set of data for each run was fit with function (2), providing $K_{\mathrm{M}, 0}$ and $\lambda_{K \mathrm{M}}$. presented in table 1 .

Table 1. Results of multiplying ratios in individual tests and averages for each multiplier.

\begin{tabular}{|c|c|c|c|c|}
\hline & \multicolumn{2}{|c|}{ Multiplier 1 } & \multicolumn{2}{c|}{ Multiplier 2 } \\
\hline & $K_{\mathrm{M}, 0}$ & $\begin{array}{c}\lambda_{K \mathrm{M}} \cdot 10^{7} / \\
\mathrm{MPa}^{-1}\end{array}$ & $K_{\mathrm{M}, 0}$ & $\begin{array}{c}\lambda_{K \mathrm{M}} \cdot 10^{7} / \\
\mathrm{MPa}^{-1}\end{array}$ \\
\hline Run 1 & 19.987668 & 3.79 & 20.008672 & 3.55 \\
\hline Run 2 & 19.989101 & 2.89 & 20.008994 & 3.39 \\
\hline Average & 19.988384 & 3.34 & 20.008833 & 3.47 \\
\hline
\end{tabular}

The performance of the multipliers has been found to be satisfactory: the $K_{\mathrm{M}}$ values were reproducible within $\pm 4 \cdot 10^{-5}$ for multiplier 1 and $\pm 2 \cdot 10^{-5}$ for multiplier 2 in relative units. Herewith and taking into account the uncertainties of the reference LP and HP piston gauges, the relative standard uncertainty of the multipliers in the pressure range (100 to $500 \mathrm{MPa}$ ) lies between (4 and $5.5) \cdot 10^{-5}$. With the same data, the relative standard uncertainty in the range (1 to 1.6) GPa can be expected to be $(1.3$ to 2$) \cdot 10^{-4}$, which is a very preliminary estimation and which must be confirmed by experiments at higher pressures. In particular, the difference between the theoretical and experimental $\lambda_{K M}$ indicates that the models need improvement.

\subsection{Material properties}

Elastic constants and hardness of pressure balance materials as well as density and viscosity of the pressure transmitting liquids had to be known for analysing the pressure multipliers and designing the pressure generation system. Elastic constants were used in the FEA of the pressure multipliers and the HP components. Knowledge of hardness of tubing, valves, connectors and sealing lenses is necessary to achieve reliable tight connections between them. Pressure dependences of the liquids' density and viscosity must be known to model the flow between piston and cylinder, to predict sufficient lubrication of the piston and cylinder and to guarantee that the liquid does not solidify at the maximum operation pressure.

\subsubsection{Elastic constants}

Tungsten carbide materials are advantageous for the PCAs due to their high elastic modulus and shape stability resulting from a purely elastic deformation under load. At the same time, the values of the elastic constants of WC materials used for PCAs are usually rather uncertain. The reason is that these materials are produced from WC powder by a high temperature sintering process using molten cobalt or nickel as a binder. The dependence on the WC-to-binder composition means that the elastic properties of a sintered material can vary from batch to batch even for a nominally identical material. This is caused by a possible inhomogeneity of the binder distribution, porosity and textures in the material. Only measurement on a specific material of a particular PCA can guarantee correctness of its elastic constants. Due to the very high Young's modulus of tungsten carbides (about 3 times higher than that of steel) and due to the usually small size of tungsten carbide samples, typically smaller than $1 \mathrm{~cm}$, classical static load machines cannot be readily used for the measurement. Instead, the RUS was applied to measure $E$ and $\mu$. In this method, the resonance frequencies of the elastic vibrations of a geometrically regular solid body are measured and the elastic constants are derived from these frequencies, dimensions of this body and its density. For the measurements, a modernised DRS Modulus II instrument manufactured by Dynamic Resonance Systems International, LLC, USA, was used. The data evaluation was performed using the algorithm developed by Migliori et al. [7, 8]. Experimental details of the method, its validation and results obtained for numerous materials are presented in $[9,10]$.

Elastic constants of steels used for the PCAs and the pressure generation system were measured using the strain gauge (SG) method described in [11] and modified in the present work. In the actual realisation of this method, strain gauge rosettes were applied to the lateral surfaces of cylindrical samples at angular positions 0,90 , 180 and $270^{\circ}$ (figure 8 ). 


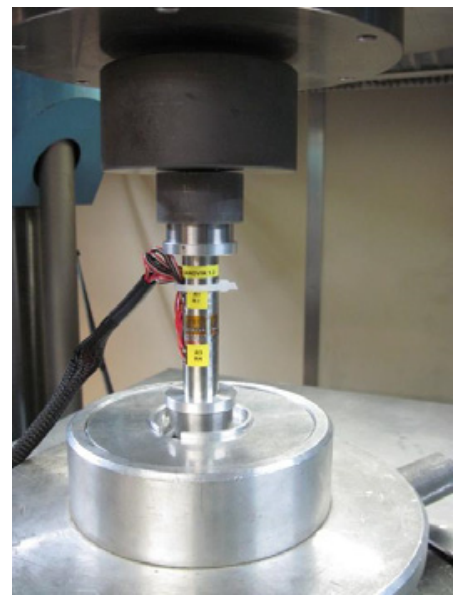

Figure 8. Cylindrical sample with strain gauge rosettes prepared for elastic constants measurements.

Each of the SG rosettes had 3 sensing elements measuring strains $(\varepsilon)$ in two orthogonal and in a respective diagonal direction. From these three strains, the principal axial $\left(\varepsilon_{1}\right)$ and tangential strains $\left(\varepsilon_{2}\right)$ could be derived. The load of the samples $(F)$ was accurately measured using a hydraulic force machine (figure 9 ).

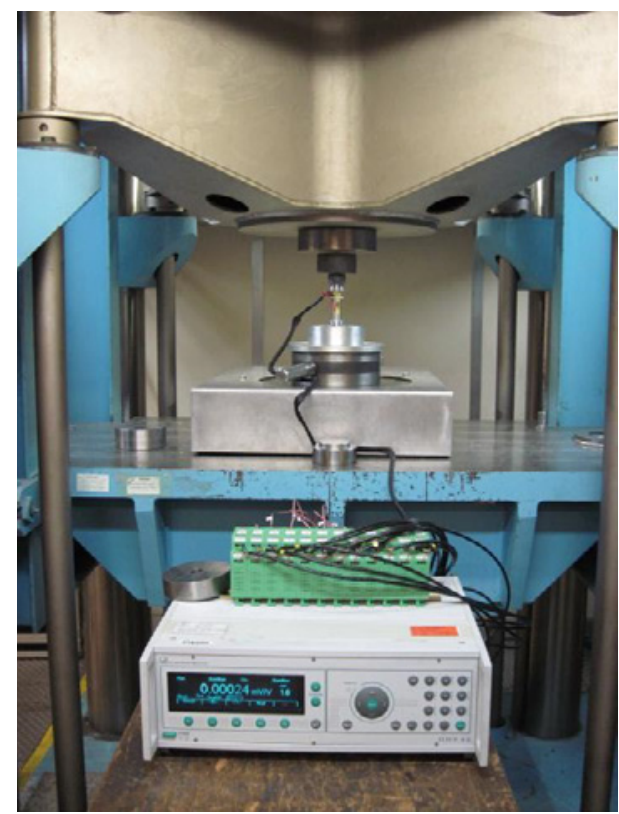

Figure 9. Arrangement of a sample in a hydraulic force machine for elastic constants measurement by the SG method.

The stresses $(\sigma)$ in the samples were determined from the load and sample's cross-sectional area $(S)$. Young's modulus was then calculated from Hooke's law $\sigma=F / S=E \cdot \varepsilon_{1}$ and Poisson's ratio from the relation $\varepsilon_{2}=-\mu \cdot \varepsilon_{1}$

Three different sorts of steel were studied:

- 30NiCrMo16 steel produced by Aubert \& Duval, France, under the name F.D.M.A (FDMA). This steel was used for the sleeves of the multipliers' LP and HP PCAs. Two cylindrical samples with an inner diameter of
$15.8 \mathrm{~mm}$, outer diameter of $30 \mathrm{~mm}$ and a length of $41 \mathrm{~mm}$ were prepared and used in the measurements.

- Austenitic steel produced by Sandvik, Sweden, under the name HP160 (HP160). It is the material used for the HP tubing. Two cylindrical samples with an inner diameter of $1.8 \mathrm{~mm}$, outer diameter of $19 \mathrm{~mm}$ and a length of $80 \mathrm{~mm}$ were prepared.

- Steel used by Harwood Engineering Company, USA, as material of their HP fittings (Harwood). An exact steel specification was unfortunately not available. One sample was manufactured from a tee and another one from an elbow connector. Each of them was a cylinder of $26 \mathrm{~mm}$ diameter and $50 \mathrm{~mm}$ length.

Results of the elastic constants' measurements are summarised in table 2.

Table 2. Elastic constants with their associated standard uncertainties of WC and steel materials measured with the RUS and the SG method.

\begin{tabular}{|c|c|c|c|}
\hline Part / Material & $E / G P a$ & $\mu$ & Method \\
\hline $\begin{array}{c}\text { LP PCA, HP cylinder / } \\
\text { WC-6\%Co }\end{array}$ & $\begin{array}{c}624.86 \\
\pm 0.16\end{array}$ & $\begin{array}{c}0.2117 \\
\pm 0.0002\end{array}$ & RUS \\
\hline HP piston / WC-10\%Co & $\begin{array}{c}577.9 \\
\pm 1.5\end{array}$ & $\begin{array}{c}0.2201 \\
\pm 0.0004\end{array}$ & RUS \\
\hline LP \& HP sleeves / & $\begin{array}{c}203.4 \\
\pm 2.7\end{array}$ & $\begin{array}{c}0.3031 \\
\pm 0.0008\end{array}$ & SG \\
\hline FDMA & $\begin{array}{c}186.3 \\
\pm 2.1\end{array}$ & $\begin{array}{c}0.3037 \\
\pm 0.0009\end{array}$ & SG \\
\hline Tubing / HP160 & $\begin{array}{c}\text { SO8.04 } \\
\pm 1.2\end{array}$ & $\begin{array}{c}0.2874 \\
\pm 0.0005\end{array}$ & SG \\
\hline Fittings / Harwood
\end{tabular}

The uncertainties reported in table 2 are sufficiently small for an FEA of the PCAs and pressure components with required accuracy. These uncertainties include uncertainties of the measurement methods and differences in the results obtained for different samples of nominally the same material. In the case of the Harwood steels, no significant difference was discovered between the materials of the tee and the elbow connectors.

\subsubsection{Hardness}

For realising leak-tight connections between the tubing and other parts of pressure balances (PCAs, lenses, connectors and valves), the hardness of the materials used is of importance in addition to the materials' elastic properties.

The Vickers hardness was measured on the following materials:

- HP components manufactured by Harwood: tube, cross connector and a sealing lens in the original state. The Harwood tube comprises two layers. Hardness of both inner and outer layers was determined.

- Tubing of HP160 produced by Sandvik. Two samples of the tube were studied. On one of them, measurements were performed near the tube bore (2) and, on the other, near the tube's exterior (3). 
- Five samples of steel 30CrNiMo8, also known under the designation D22S, which is widely used in HP applications. Two of the samples were sealing lenses in the original unloaded state (1) and (2); one was a used lens (3); and one was a plane drilled sample, which was measured (4), re-polished after the first measurement and then measured again (5).

- Two sealing lenses made of steel X5CrNiCuNb16-4 manufactured by HBM, Germany, intended for $1.5 \mathrm{GPa}$ applications, one in the original state (1) and the other having been preloaded (2).

- A sealing lens manufactured by Maximator, Germany, intended for $1.4 \mathrm{GPa}$ applications, in the original state.

Results of the hardness measurements are presented in figure 9 .

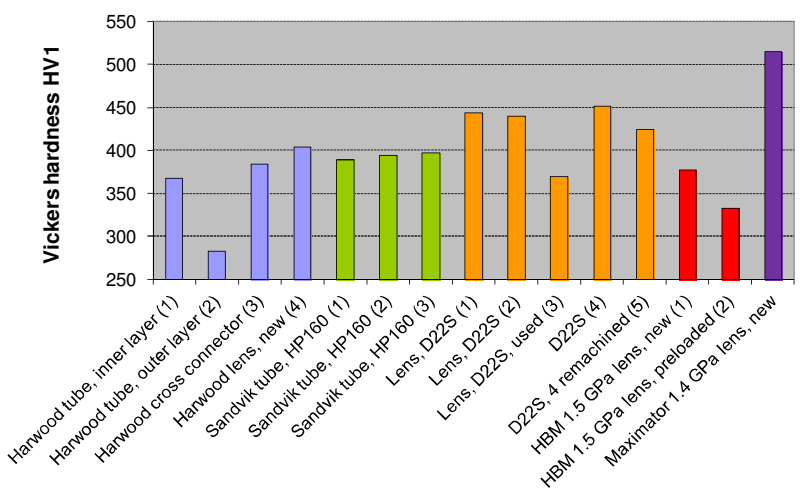

Figure 9. Hardness of HP components.

The inner layer of the Harwood tube has a significantly higher hardness than the outer layer. Such a hardness relation in the two layers appears to be nonoptimal. Under pressure, the inner layer undergoes higher deformations than the outer one and, therefore, a material with a higher elongation limit, i.e. higher ductility and lower hardness, would be better for the inner layer. The hardness of the Harwood cross connector is higher than that of the Harwood tube. This is favourable for a direct connection of the tube to the connector because such a hardness relation prevents damage of the connector's seats. Vice versa, the highest hardness of the Harwood lens can damage the tube and the connector seats.

The measurements on the HP160 samples demonstrate high homogeneity of the Sandvik tube.

Material D22S shows a wide scatter of hardness which evidently comes from different thermal treatment of the samples. The hardness of D22S appears to be nonuniform and/or to change with mechanical treatment of the material. Evidently, the drill processing of sample 4 caused its hardness to become greater. After the sample re-polishing, the hardened level was removed and the hardness decreased.

For $\mathrm{X} 5 \mathrm{CrNiCuNb} 16-4$ material of the lenses produced by HBM, the hardness is dependent on mechanical treatment. After loading the lens, its hardness became lower.

The lenses by Maximator have the highest hardness among the studied materials. The measured hardness corresponds to a theoretical tensile strength of $1.75 \mathrm{GPa}$. However, that high hardness can cause damage when the lens is used with HP160 tubes.

For the hardness values measured on different HP parts, D22S steal after appropriate thermal treatment, appears as a suitable material for the sealing lenses.

\subsubsection{Liquid density and viscosity}

DHS is a liquid widely used in pressure balances up to $1 \mathrm{GPa}$. Its density $(\rho)$ and dynamic viscosity $(\eta)$ in dependence on pressure were measured in the pressure range up to $1 \mathrm{GPa}$ at temperatures $(0,25,37.78){ }^{\circ} \mathrm{C}$ with an uncertainty $\geq 3 \%$ in [12] and in the pressure range up to $500 \mathrm{MPa}$ at $20{ }^{\circ} \mathrm{C}$ with an uncertainty of $1 \%$ in [13]. On the basis of that experimental data $\rho$ and $\eta$ at $20{ }^{\circ} \mathrm{C}$ were presented in [14] by equations for $p \leq 500 \mathrm{MPa}$ :

$$
\begin{aligned}
\rho /\left(\mathrm{kg} / \mathrm{m}^{3}\right)= & 912.6657+0.752097 \cdot p \\
& -1.64485 \cdot 10^{-3} \cdot p^{2}+1.45625 \cdot 10^{-6} \cdot p^{3}, \\
\eta /(\mathrm{Pa} \cdot \mathrm{s})= & 0.021554 \cdot\left(1+1.90036 \cdot 10^{-3} \cdot p\right)^{8.8101},
\end{aligned}
$$

and for $500 \mathrm{MPa}<p \leq 1 \mathrm{GPa}$ :

$$
\begin{aligned}
\rho /\left(\mathrm{kg} / \mathrm{m}^{3}\right)= & 915.61+0.505727 \cdot p-0.661573 \cdot 10^{-3} \cdot p^{2} \\
& +0.584283 \cdot 10^{-6} \cdot p^{3}-0.204436 \cdot 10^{-9} \cdot p^{4}, \quad(6) \\
\eta /(\mathrm{Pa} \cdot \mathrm{s})= & 469.968-4.93208 \cdot p+0.0213348 \cdot p^{2} \\
& -4.8768 \cdot 10^{-5} \cdot p^{3}+6.25155 \cdot 10^{-8} \cdot p^{4} \\
& -4.28033 \cdot 10^{-11} \cdot p^{5}+1.2575 \cdot 10^{-14} \cdot p^{6},
\end{aligned}
$$

where $p$ is given in MPa. The experimental and the model equations' data are presented in figures 10 and 11 . In figure 11 , the viscosity range limits acceptable for proper operation of a pressure balance are shown by the dashed lines. At pressures $>1 \mathrm{GPa}$ the viscosity of DHS exceeds the acceptable range.

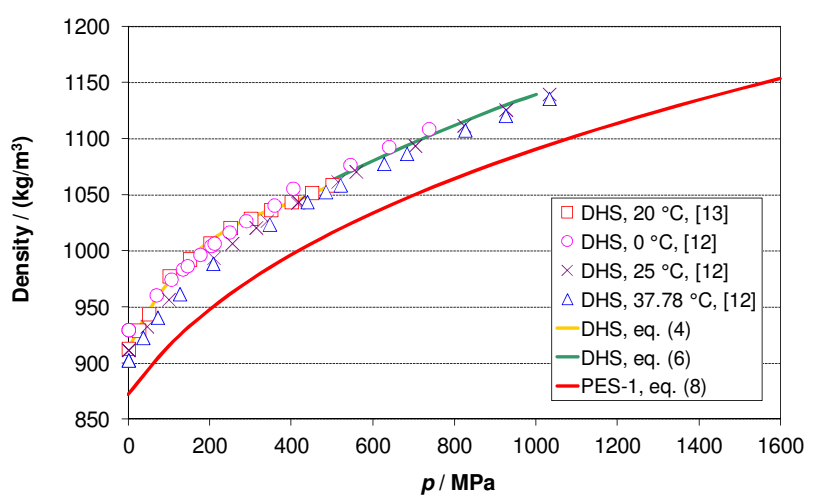

Figure 10. Density vs. pressure of DHS and PES-1.

For PES- 1 its $\rho$ and $\eta$ were determined in the pressure range up to $700 \mathrm{MPa}$ in [3]. The density at $20{ }^{\circ} \mathrm{C}$ can be described with a good approximation by equation

$$
\rho /\left(\mathrm{kg} / \mathrm{m}^{3}\right)=872.5 /\left[1-0.106 \cdot \ln \left(1+5.59 \cdot 10^{-3} \cdot p\right)\right],
$$




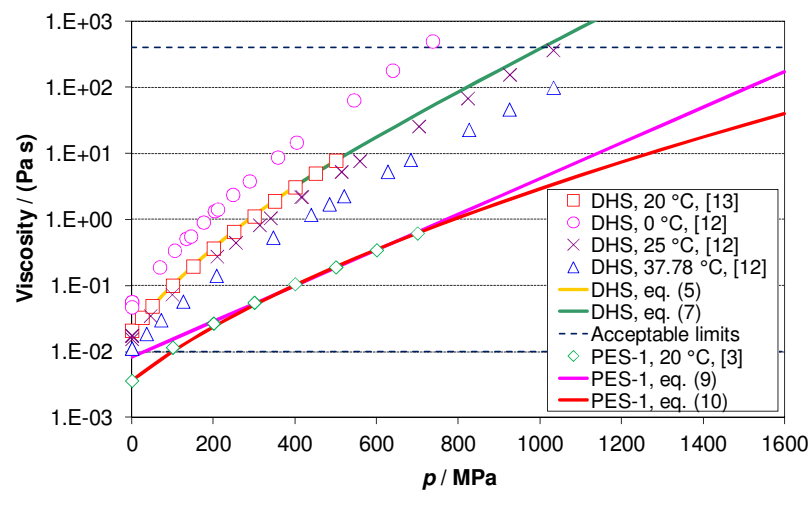

Figure 11. Viscosity vs. pressure of DHS and PES-1.

and the experimental viscosity at $20{ }^{\circ} \mathrm{C}$ can be approximated by Barus':

$$
\eta /(\mathrm{Pa} \cdot \mathrm{s})=8.30 \cdot 10^{-3} \cdot \exp (0.00622 \cdot p)
$$

or Roelands' equation:

$$
\eta /(\mathrm{Pa} \cdot \mathrm{s})=3.65 \cdot 10^{-3} \cdot \exp \left\{4.1 \cdot\left[\left(1+5.1 \cdot 10^{-3} \cdot p\right)^{0.54}-1\right]\right\},(10)
$$

with $p$ to be taken in MPa. The experimental data for PES-1 and the model approximations are included in figures 10 and 11 . The extrapolations of $\eta /(p)$ beyond $700 \mathrm{MPa}$ with Barus' and Roelands' equations differ increasingly with increasing pressure. However, both dependences stay in the acceptable viscosity range even at the maximum pressure of $1.6 \mathrm{GPa}$.

To study the stability of the PES-1 properties, its density and viscosity were accurately measured at atmospheric pressure on three different samples, one of which had several times been subjected to pressures of more than $1.6 \mathrm{GPa}$. The results of these measurements are presented in table 3 .

Table 3. Density $(\rho)$ and viscosity $(\eta)$ of PES- 1 at atmospheric pressure and different temperatures $(t)$.

\begin{tabular}{|c|c|c|c|}
\hline Sample & $t /{ }^{\circ} \mathrm{C}$ & $\rho /\left(\mathrm{g} / \mathrm{cm}^{3}\right)$ & $\eta /(\mathrm{mPa} \cdot \mathrm{s})$ \\
\hline & 18 & 0.87384 & 3.357 \\
1, unloaded & 20 & 0.87237 & 3.226 \\
& 22 & 0.87089 & 3.107 \\
\hline \multirow{2}{*}{ 2, unloaded } & 18 & 0.87390 & 3.370 \\
& 20 & 0.87242 & 3.241 \\
3, preloaded with & 18 & 0.87094 & 3.120 \\
$p \geq 1.6 \mathrm{GPa}$ & 22 & 0.87285 & 3.404 \\
& 22 & 0.87137 & 3.271 \\
\hline \multirow{2}{*}{ Standard uncertainty } & 0.01 & 0.00001 & $0.5 \%$ \\
\hline
\end{tabular}

The density and viscosity of the unloaded samples 1 and 2 differ relatively by $7 \cdot 10^{-5}$ and $5 \cdot 10^{-3}$, respectively. Preloading with 1.6 GPa causes relative changes in the density and viscosity of $5 \cdot 10^{-4}$ and $9 \cdot 10^{-3}$, respectively. These changes appear as a result of the liquid compressing, but they can also be caused by liquid contamination in the pressure intensifier. In any case, the observed changes are sufficiently low to consider PES-1 to be stable enough against the pressurization.

\subsection{Transfer standard}

An HP transfer standard was realised on the basis of high-precision $0.5 \mathrm{GPa}, 1 \mathrm{GPa}$ and $1.5 \mathrm{GPa}$ pressure transducers and is to be circulated among PTB, CMI, SMU, METAS and LNE.

The aim is to determine the applicability of highpressure transducers as the TS for pressure comparisons. Moreover, the performance of the underlying new 1.5 GPa transducers and, in particular, the correlation between the maximum pressure range and performance for different types of high pressure transducers are to be investigated. Typical parameters of the transducers such as drift, hysteresis, sensitivity, repeatability, long-term stability and load cycling effects are to be studied and optimal calibration procedures determined. In addition, it is expected that the measurements at the NMIs will furnish information about the equivalence of their pressure standards.

The structure of the TS is shown in figure 12.
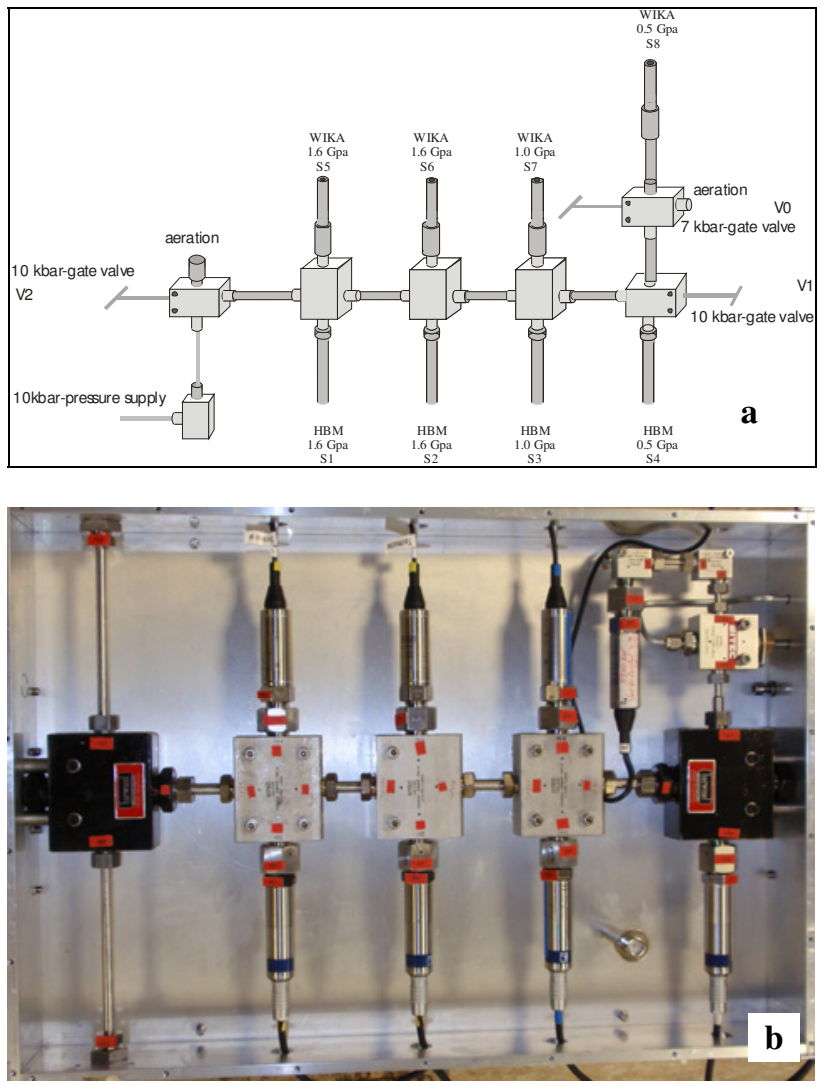

Figure 12. Block diagram (a) and appearance (b) of transfer standard.

The TS includes four pressure transducers with the ranges $0.5 \mathrm{GPa}, 1.0 \mathrm{GPa}$ and $1.5 \mathrm{GPa}$ (2 pieces) manufactured by HBM, Germany, and four pressure transducers with the same pressure ranges produced by WIKA, Germany.

The HBM pressure transducers are of type 1-P3TCP, use foil strain gauges as a sensing element and have a nominal sensitivity of $1 \mathrm{mV} / \mathrm{V}$ over the measurement 
range. They are operated with an HBM measuring amplifier model DMP40S2.

The WIKA pressure transducers are of type HP-2-S, use thin-layer strain gauges as a sensing element and have a resolution of $1 \mathrm{kPa}$. They can be directly read out via USB using appropriate software.

Special software was developed for monitoring all the eight pressure transducers of the TS, and a technical protocol of the measurements was prepared. Therein, details of the measurements and the measurement procedure as shown in figure 13 are specified.

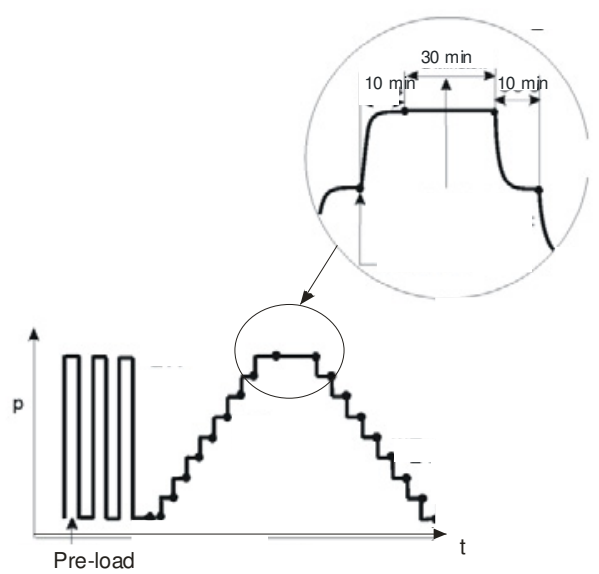

Figure 13. Measurement procedure for TS.

Preparation of the pressure transducers and an initial investigation of the TS behaviour were performed at PTB. In figure 14 , the readings of one of the $1.5 \mathrm{GPa}$ pressure transducers are shown when it is preloaded with a pressure of about $1.6 \mathrm{GPa}$.

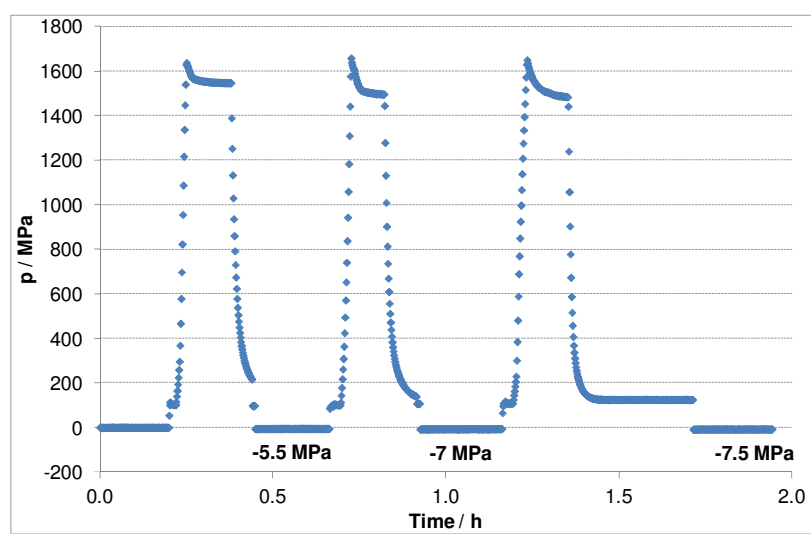

Figure 14. Output of a 1.5 GPa pressure transducer when preloading it with a pressure of $1.6 \mathrm{GPa}$.

The preloads were performed with the aid of a $2 \mathrm{GPa}$ pressure intensifier, in which only the pressure on the low pressure side could be controlled. The pressure drops indicated by the transducer after reaching the maximum are evidently caused by some leakage in the hydraulic system. At that stage it was not possible to stabilise and to accurately measure the pressure of $1.6 \mathrm{GPa}$. From figure 14 it can be seen that the first preload of the transducer caused a change of its zero point of about
-5.5 MPa. Two subsequent loads with 1.6 GPa slightly increased this shift to $-7 \mathrm{MPa}$ and then to $-7.5 \mathrm{MPa}$. At this state, the transducer is expected to be stabilised if afterwards it is used for measurements only up to $1.5 \mathrm{GPa}$, which should be confirmed by future measurements. After the initial investigation, the TS is used in the comparison which is in progress.

\section{Summary and outlook}

The differences between the experimental and theoretical pressure distortion coefficients of the $1.6 \mathrm{GPa}$ multipliers can be associated with the fact that only approximate information about the cylinder bore shape in the outlet area of the HP PCAs was included in the FEA model. In addition, only estimated dimensional data on the conical shape of the sliding sleeves of the LP PCAs were available and used in the analysis so far. Complete dimensional measurements on both piston and cylinder bore of the HP PCAs and the sliding sleeves of the LP PCAs will be carried out in the future and their results will be used in the FEA.

Currently, the pressure dependences of the density and viscosity of PES-1 are known from experiments carried out in the range up to $700 \mathrm{MPa}$. Extrapolation using model equations suggests that the viscosity lies in the reasonable range over a whole pressure range up to 1.6 GPa. However, an anomaly of the viscosity or even the liquid's solidification with increasing pressure cannot be generally excluded. Experiments to measure the density and viscosity of PES-1 at pressures up to at least $1.4 \mathrm{GPa}$ are planned at the Technical University Clausthal for the near future.

In this stage of the comparison, measurements have been successfully completed at PTB and CMI. Extensive experimental data on the pressure transducers which will be collected by the end of the comparison will allow the manufacturers to improve the design of the pressure transducers and should make possible the selection of optimal procedures for the calibration of high pressure transducers.

\section{Acknowledgements}

We acknowledge contributions by R. Haines, P. Delajoud, F. Valenzuela and M. Bair (Fluke) to the development and characterisation of the $1.6 \mathrm{GPa}$ multipliers. We very much appreciate the contributions of PTB's Pressure Working Group staff A. Ahmed to the elastic constants' measurements, and A. Gluschko, T. Konczak and J. Könemann to the development and characterisation of the HP transfer standard. Our thanks go to O. Jusko for performing the dimensional measurements, H. Wolf for the liquid density and viscosity measurements, as well as K. Herrmann and F. Menelao for hardness measurements (all PTB). Finally, we would like to thank the companies HBM and WIKA for providing us with the HP pressure transducers for the transfer standard. This research was carried out within the EMRP. It is jointly funded by the EMRP participating countries within EURAMET and the European Union. 


\section{References}

1. EURAMET, "High pressure metrology for industrial applications", Publishable JRP summary report for IND03 HighPRES, http://www.euramet.org/index.php?id=emrp_call_2010 2. EMRP JRP IND03 HighPRES, http://emrphighpres.cmi.cz/

3. V.M. Borovkov, Researches in the area of high pressures, 5-77 (Izdatelstvo standartov, Moscow, 1987, russ.)

4. P. Delajoud, BIPM Monographie 89/1, 114-124 (1989)

5. W. Sabuga, R. Haines, Proc. of XX IMEKO World Congress, Busan, Republic of Korea (2012), www.imeko.org/publications/wc-2012/IMEKO-WC-

2012-TC16-O1.pdf

6. W. Sabuga et al., Metrologia, 43, 311-325 (2006)

7. A. Migliori, J.L. Sarrao, Resonant ultrasound spectroscopy (Wiley, New York, 1997)

8. A. Migliori, J.D. Maynard, Rev. Sci. Instrum., 76, 1-7 (2005)

9. W. Sabuga, P. Ulbig, A.D. Salama, Proc. of Int. Metrology Conf. CAFMET-2010, Cairo, Egypt (2010)

10. A.D. Salama, W. Sabuga, P. Ulbig, Measurement, 45, 2472-2475 (2012)

11. W. Sabuga, Proc. of $17^{\text {th }}$ Int. Conf. on Force, Mass, Torque and Pressure Measurements, IMEKO TC3, Istanbul, Turkey (2001)

12. ASME Report, Viscosity and density of over 40 lubricating fluids of known composition at pressures to 150000 psi and temperatures to $425 F$ (ASME, New York, 1953)

13. P. Vergne, High Temperatures - High Pressures, 22, 613-621 (1990)

14. G.F. Molinar, Rapporto Tecnico Interno R 486 (CNR-IMGC, Torino, 1998) 